\title{
NOTE ON A CERTAIN TYPE OF PARABOLA*
}

\author{
BY W. C. GRAUSTEIN
}

In the development of the geometry of the complex domain, there has been a tendency to pass over problems of a relatively elementary character, despite their central position and importance. It is the purpose of this note to call attention to one of these problems, namely that of the classification of complex conics with respect to the group of complex rigid motions. A systematic study of this problem brings to light two special types of non-degenerate conics:the non-degenerate central conics which contain just one circular point at infinity, and the non-degenerate parabolas which are tangent to the line at infinity at a circular point. These special conics have already been considered, from a different point of view, in this Bulletin. $\dagger$

It is not our intention to go into detail, either in connection with the general problem of classification or in consideration of the special conics. We propose merely to discuss certain particularly striking properties of the special parabolas.

It is evident that the complex conic

$$
A x^{2}+B x y+C y^{2}+D x+E y+F=0,
$$

$A, B, C$ not all zero, is a special parabola if and only if

$$
B^{2}-4 A C=0, \quad A+C=0, \quad \Delta \neq 0,
$$

where $\Delta$ is the discriminant of (1).

Since a special parabola has neither focus nor directrix, the question of its eccentricity cannot be handled directly. We may, however, establish an equation determining the

* Presented to the Society, April 6, 1928.

$\dagger$ E. V. Huntington and J. K. Whittemore, Some curious properties of conics touching the line at infinity at one of the circular points, vol. 8 (190102), pp. 122-124. 
eccentricities of the general conic (1) and extend its validity to all conics. An equation with this property, whose roots are the squares of the eccentricities, is

$$
\left(B^{2}-4 A C\right) s^{2}-4\left[(A+C)^{2}+B^{2}-4 A C\right](s-1)=0 .
$$

For a special parabola the coefficients in this equation are all zero. Hence the homogeneous functions of degree zero in $A, B, C$, which for the general conic are the squares of the eccentricities, are, for a special parabola, undefined.

Theorem 1. A special parabola has no eccentricity* and is the only conic with this property.

Consider a special parabola tangent to the line at infinity at $J:(1,-i, 0)$. Let the parabola be referred to the point of contact, $O$, of the isotropic tangent through $I:(1, i, 0)$ as origin of a system of rectangular Cartesian coordinates. Then regardless of the choice of axes of coordinates, the equation of the parabola takes the form

$$
(x-i y)^{2}=2 k(x+i y), \quad k \neq 0,
$$

or, in terms of isotropic coordinates, the form

$$
\bar{z}^{2}=2 k z, \quad k \neq 0 .
$$

The rotation about $O$ through the angle $\theta$ :

$$
z^{\prime}=e^{\theta i} z, \quad \bar{z}^{\prime}=e^{-\theta i \bar{z}}
$$

carries the parabola (3) into $\bar{z}^{\prime 2}=2 k e^{-3 \theta i} z^{\prime}$ and hence, when $3 \theta \equiv 0(\bmod 2 \pi)$, into itself. Herewith, we have the most bizarre of peculiarities imaginable for a conic.

Theorem 2. A special parabola has rotational symmetry about the point of contact $O$ of its unique isotropic tangent: there exists a group of three rotations about $O$ which carry it

* It is evident that a variable conic may be made to approach a special parabola as a limit in such a way that the square of one of its eccentricities approaches any preassigned value, 0 not excluded. Herein we have, for example, the explanation of the presence of two special parabolas in a pencil of equilateral hyperbolas. See Huntington and Whittemore, loc. cit. 
into itself, namely the rotations through $0,2 \pi / 3$, and $4 \pi / 3$ radians.

It follows that there is an infinity of equilateral triangles inscribed in the parabola, all of which have the point $O$ as median or central point. Every point of the parabola, except $O$ and the point at infinity, is a vertex of just one of the triangles.

By means of equations (4), interpreted as a rotation of the axes of coordinates, equation (3) of our special parabola may be reduced to the normal form

$$
\bar{z}^{2}=2 z \text {. }
$$

It suffices to choose $\theta$ so that $e^{3 \theta i}=k$. Hence :

THEOREM 3. There are only two types of special parabolas: every two special parabolas with the same point at infinity are equivalent with respect to the group of complex rigid motions.

Here, again, the special parabolas differ from the ordinary ones. The ordinary nondegenerate parabolas tangent to the line at infinity at a given (noncircular) point are of an infinity of types depending on one complex parameter. The parameter may be taken as the square of the semi latus rectum, whose value for an ordinary nondegenerate parabola (1) is given by an expression, $\Delta /(A+C)^{3}$, which is undefined (infinite) for every special parabola.

An instructive side-light on Theorem 2 is obtained by studying its projective generalization. Consider a complex collineation of general type-of which a rotation is a special metric case - and write its equations in the canonical forms

$$
x_{1}^{\prime}=a_{1} x_{1}, \quad x_{2}^{\prime}=a_{2} x_{2}, \quad x_{3}^{\prime}=a_{3} x_{3},
$$

where $a_{1}, a_{2}, a_{3}$ are complex numbers, no two equal and no one zero. If the collineation leaves fixed a nondegenerate conic, it establishes on the conic a projectivity which is readily shown to be nonparabolic. The conic must, therefore, be tangent to two fixed lines of the collineation at two fixed 
points; that is, belong to one of the three pencils of conics

$$
k_{1} x_{1}^{2}=2 l_{1} x_{2} x_{3}, \quad k_{2} x_{2}^{2}=2 l_{2} x_{3} x_{1}, \quad k_{3} x_{3}^{2}=2 l_{3} x_{1} x_{2} .
$$

The degenerate conics of these pencils are obviously invariant with respect to the collineation. Hence, if one of the nondegenerate conics of a pencil is invariant, all the conics of the pencil are invariant. The conditions for invariance in the three cases are respectively

$$
a_{1}^{2}=a_{2} a_{3}, \quad a_{2}^{2}=a_{3} a_{1}, \quad a_{3}^{2}=a_{1} a_{2} .
$$

Any one of these relations is a consequence of the other two. The collineations (6) determined by them are

$$
\begin{aligned}
& x_{1}^{\prime}=\omega^{2} x_{1}, x_{2}^{\prime}=\omega x_{2}, x_{3}^{\prime}=x_{3} ; \\
& x_{1}^{\prime}=\omega x_{1}, \quad x_{2}^{\prime}=\omega^{2} x_{2}, x_{3}^{\prime}=x_{3},
\end{aligned}
$$

where $\omega$ is a primitive cube root of unity.

THEOREM 4. The only collineations of general type (6) which leave fixed each of the conics of two, and hence of all three, of the pencils of conics (7) are the collineations (8).

If two of the three fixed points of the collineation (6) are the circular points at infinity, two of the pencils (7) are those of the special parabolas having the odd fixed point as the point of contact of their unique isotropic tangents, the third pencil is that of the circles with the odd fixed point as center, and the two collineations (8) are the rotations about the odd fixed point through $2 \pi / 3$ and $4 \pi / 3$ radians.

One last remark. A real nondegenerate conic (1) is a circle if and only if

$$
(A+C)^{2}+B^{2}-4 A C=0 \text { or }(A-C)^{2}+B^{2}=0 .
$$

But the complex nondegenerate conics (1) defined by this relation include, besides the circles, the special parabolas and the special central conics. Moreover, both eccentricities of a special central conic, as well as both those of a circle, are zero.

HaRVARd University 